\title{
Design of experiments and optimization of composite structures using solid-shell elements
}

\author{
M. Hannachi ${ }^{1}$, H. Naceur ${ }^{1}$, J. L. Batoz ${ }^{1} \&$ S. Belouettar ${ }^{2}$ \\ ${ }^{1}$ University of Technology of Compiègne, Compiègne, France \\ ${ }^{2}$ Public Research Center Henri Tudor, Luxembourg
}

\begin{abstract}
The present study investigates the formulation and use of solid-shell finite element models which are able to model thin and thick, isotropic and multilayered composite structures using a purely 3D formulation based only on displacement dof. The second part of the paper deals with the optimization of composite structures, using a specific response surface method. This technique has been shown to be more efficient than classical gradient based methods. Several applications combining our solid-shell finite element model and the specific response surface method are shown in order to assess current procedure capabilities.
\end{abstract}

\section{Introduction}

Solid-shell elements have many advantages compared to the degenerated shell models, because of their kinematic simplicity, their ability in modeling industrial structures generally composed of bulk and thin-walled regions and also special rotations treatment in geometric nonlinear analysis can be avoided. Unfortunately, the formulation of valid solid-shell elements is more complicated than the one used for degenerated shell elements since solid-shell elements are bothered by membrane, shear, trapezoidal and thickness lockings [2,3].

During last five years, Response Surface Methods (RSM) have gained more and more importance in the optimization of general shell structures [5]. RSM has the advantage of replacing a complex response model by an approximate one based on results calculated at various points in the design space. The optimization is then performed at a lower cost over such response surfaces. Two important issues when applying RSM to a particular problem concern the design of experiments 
and construction of accurate function approximations so that rapid convergence may be achieved. In the present work, we exploit the RSM based on Diffuse approximation (DA) and particularly the notion of pseudo-derivative to design a specific optimization technique, custom built for this regression model. This new method is an extension of pattern search in two aspects: (1) accommodation of arbitrary regular and irregular patterns; (2) design points eligible for inclusion in any pattern instance belong to a predefined set in the design space where experiments are authorized.

In the final section of the paper, we present numerical applications combining a solid-shell finite element models for the modeling of Multilayered composite structures with our response surface method.

\section{FE formulation of the eight-node hexahedron}

\subsection{Kinematics of solid-shell models}

In this section, the formulation of the eight-node solid-shell element is briefly recalled. With respect to nodal designation (Figure 1), the coordinate vector $\mathbf{X}$ and displacement vector $\mathbf{U}_{\mathbf{q}}$ of the element are

$$
\begin{aligned}
& \mathbf{X}_{\mathbf{q}}=\mathbf{X}_{\mathbf{0}}(\xi, \eta)+\zeta \mathbf{X}_{\mathbf{n}}(\xi, \eta)=\sum_{i=1}^{4} N_{i}(\xi, \eta)\left(\frac{1-\zeta}{2} \mathbf{X}_{\mathbf{i}}^{-}+\frac{1+\zeta}{2} \mathbf{X}_{\mathbf{i}}^{+}\right) \\
& \mathbf{U}_{\mathbf{q}}=\mathbf{U}_{\mathbf{0}}(\xi, \eta)+\zeta \mathbf{U}_{\mathbf{n}}(\xi, \eta)=\sum_{i=1}^{4} N_{i}(\xi, \eta)\left(\frac{1-\zeta}{2} \mathbf{U}_{\mathbf{i}}^{-}+\frac{1+\zeta}{2} \mathbf{U}_{\mathbf{i}}^{+}\right)
\end{aligned}
$$

where $N_{i}$ are the two-dimensional eight-node Lagrangian interpolation functions, $\mathbf{X}_{\mathbf{i}}^{-}, \mathbf{U}_{\mathbf{i}}^{-}$and $\mathbf{X}_{\mathbf{i}}^{+}, \mathbf{U}_{\mathbf{i}}^{+}$are respectively, the coordinate and displacement vectors of the $\mathrm{i}^{\text {th }}$ node on the bottom and top shell surfaces (Figure 1).

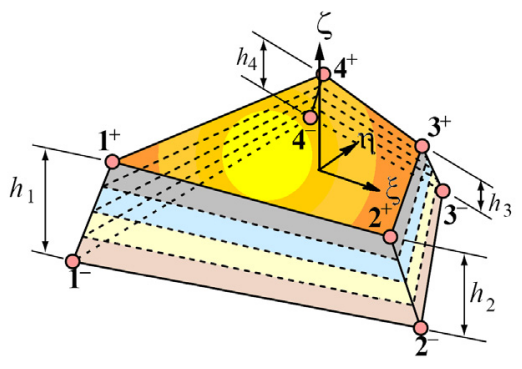

Figure 1: Eight-node solid-shell element.

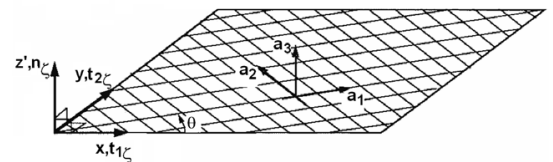

Figure 2: Fiber-reinforced lamina orientation axes. 
The infinitesimal covariant element strains with respect to the parametric coordinates are

$$
\begin{gathered}
\varepsilon_{\xi \xi}=\mathbf{X}_{, \xi}^{\mathbf{T}} \mathbf{U}_{, \xi} ; \quad \varepsilon_{\eta \eta}=\mathbf{X}_{, \eta}^{\mathbf{T}} \mathbf{U}_{, \eta} ; \quad \gamma_{\xi \eta}=\mathbf{X}_{, \xi}^{\mathbf{T}} \mathbf{U}_{, \eta}+\mathbf{X}_{, \eta}^{\mathbf{T}} \mathbf{U}_{, \xi} \\
\varepsilon_{\zeta \zeta}=\mathbf{X}_{, \zeta}^{\mathbf{T}} \mathbf{U}_{, \zeta} ; \quad \gamma_{\xi \zeta}=\mathbf{X}_{, \xi}^{\mathbf{T}} \mathbf{U}_{, \zeta}+\mathbf{X}_{, \zeta}^{\mathbf{T}} \mathbf{U}_{, \xi} ; \quad \gamma_{\eta \zeta}=\mathbf{X}_{, \eta}^{\mathbf{T}} \mathbf{U}_{, \zeta}+\mathbf{X}_{, \zeta}^{\mathbf{T}} \mathbf{U}_{, \eta}
\end{gathered}
$$

\subsection{Material law in convected basis}

The constitutive relation of laminated composites can be described by using an orthotropic material law. For that purpose, we express the components of the tangent elastic moduli tensor relative to the fiber reference axis $\left\{\mathbf{a}_{1} ; \mathbf{a}_{2} ; \mathbf{a}_{3}\right\}$ of a lamina.

$$
\mathbf{C}=\left[\begin{array}{cccccc}
C^{1111} & C^{1112} & 0 & C^{1113} & 0 & 0 \\
C^{1122} & C^{2222} & 0 & C^{2223} & 0 & 0 \\
0 & 0 & C^{1212} & 0 & 0 & 0 \\
C^{1133} & C^{2233} & 0 & C^{3333} & 0 & 0 \\
0 & 0 & 0 & 0 & C^{1313} & 0 \\
0 & 0 & 0 & 0 & 0 & C^{2323}
\end{array}\right]
$$

where the components $C^{i j k l}$ take the following expressions: $C^{1111}=E_{1}(1-$ $\left.v_{23} v_{32}\right) / K, C^{1122}=E_{1}\left(v_{21}+v_{23}\right) / K, C^{2222}=E_{2}\left(1-v_{13} v_{31}\right) / K, C^{1113}=$ $E_{1}\left(v_{31}+v_{21} v_{32}\right) / K, C^{2223}=E_{2}\left(v_{32}+v_{12} v_{31}\right) / K, C^{3333}=E_{3}\left(1-v_{12} v_{21}\right) / K$, $C^{1212}=G_{12}, C^{1313}=G_{13}, C^{2323}=G_{23}, K=1-v_{12} v_{21}-v_{13} v_{31},-v_{23} v_{32}-$ $2 v_{12} v_{23} v_{31} . \nu_{i j} E_{j}=\nu_{j i} E_{i}$, for $(i, j=1,2,3$ with $i \neq j)$ and $E_{1}, E_{2}, E_{3}$ are the Young's moduli in the principal material directions $\left\{\mathbf{a}_{\mathbf{1}} ; \mathbf{a}_{\mathbf{2}} ; \mathbf{a}_{\mathbf{3}}\right\}$, respectively, and $\nu_{i j}$ and $G_{i j}$ the Poisson's ratio and the shear modulus respectively.

Since matrix $\mathbf{C}$ is associated with the principal material directions, we need to transform it from the lamina coordinate axes $\left\{\mathbf{a}_{1} ; \mathbf{a}_{2} ; \mathbf{a}_{3}\right\}$ to the global Cartesian coordinate axes $\left\{\mathbf{e}_{\mathbf{1}} ; \mathbf{e}_{\mathbf{2}} ; \mathbf{e}_{\mathbf{3}}\right\}$. With $\theta$ being the fiber orientation angle relative to the global Cartesian system (see Figure 2), the relationship between the lamina coordinate system and the global Cartesian coordinate system is given by

$$
\mathbf{a}_{1}=\cos \theta \mathbf{e}_{1}+\sin \theta \mathbf{e}_{\mathbf{2}}, \quad \mathbf{a}_{\mathbf{2}}=-\sin \theta \mathbf{e}_{\mathbf{1}}+\cos \theta \mathbf{e}_{\mathbf{2}}, \quad \mathbf{a}_{\mathbf{3}}=\mathbf{e}_{\mathbf{3}}
$$

The final constitutive tensor $\mathbf{C}$ can be expressed in the convective coordinates as

$$
\tilde{\mathbf{C}}=\mathbf{T}_{\mathbf{G}}^{\mathbf{T}} \mathbf{C} \mathbf{T}_{\mathbf{G}}
$$

\subsection{Principal of virtual work and stiffness matrix calculation}

In order to deal with the several lockings separately, we need to separate the expression of virtual internal work by uncoupling the membrane/bending, 
thickness and transverse shear [1,2].

$$
\begin{gathered}
W_{i n t}=W_{i n t}^{m f}+W_{i n t}^{e z}+W_{i n t}^{c} \\
W_{i n t}^{m f}=\int_{V} \delta \varepsilon_{s}^{T} \tilde{\mathbf{C}}_{\mathbf{1}} \varepsilon_{s} d v=\delta \mathbf{u}_{n}^{T} \mathbf{K}_{m f} \mathbf{u}_{n} ; \quad \mathbf{K}_{m f}=\int_{v} \mathbf{B}_{m f}^{T} \tilde{\mathbf{C}}_{\mathbf{1}} \mathbf{B}_{m f} d v \\
W_{i n t}^{e z}=\int_{V} \delta \varepsilon_{z}^{T} \tilde{\mathbf{C}}_{\mathbf{2}} \varepsilon_{z} d v=\delta \mathbf{u}_{n}^{T} \mathbf{K}_{e z} \mathbf{u}_{n} ; \quad \mathbf{K}_{e z}=\int_{v} \mathbf{B}_{z}^{T} \tilde{\mathbf{C}}_{\mathbf{2}} \mathbf{B}_{z} d v \\
W_{i n t}^{c}=\int_{V} \delta \gamma_{s}^{T} \tilde{\mathbf{C}}_{\mathbf{3}} \gamma_{s} d v=\delta \mathbf{u}_{n}^{T} \mathbf{K}_{c} \mathbf{u}_{n} ; \quad \mathbf{K}_{c}=\int_{v} \mathbf{B}_{c}^{T} \tilde{\mathbf{C}}_{\mathbf{3}} \mathbf{B}_{c} d v
\end{gathered}
$$

and $\varepsilon_{\mathbf{s}}^{T}=<\varepsilon_{x x} \varepsilon_{y y} \gamma_{x y}>, \varepsilon_{\mathbf{z}}^{T}=<\varepsilon_{x x} \varepsilon_{y y} \varepsilon_{z z}>, \gamma_{\mathbf{s}}^{T}=<\gamma_{x z} \gamma_{y z}>$

A solid-shell element formulated using equations (9), (10), (11) with standard integration based on a $2 \times 2 \times 2$ Gauss schema will fail because of numerous locking phenomena.

\subsubsection{Remedies for shear locking}

An effective method of resolving shear locking is the Assumed Natural Strain method in which the natural transverse shear strains are sampled and then interpolated at some discrete element points. The transverse shear strains $\gamma_{\xi \zeta}$ and $\gamma_{\eta \zeta}$ are calculated according to the average surface plan $(\zeta=0)$, assuming that they vary linearly, and are function of $\gamma_{\xi}$ and $\gamma_{\eta}$ at the mid-side points:

$$
\gamma_{\xi \zeta}^{A N S}=\frac{1-\eta}{2} \gamma_{\xi}^{A 1}+\frac{1+\eta}{2} \gamma_{\xi}^{A 2} ; \quad \gamma_{\eta \zeta}^{A N S}=\frac{1-\xi}{2} \gamma_{\eta}^{B 1}+\frac{1+\xi}{2} \gamma_{\eta}^{B 2}
$$

\subsubsection{Remedies for thickness and trapezoidal lockings}

Similar to shear locking, trapezoidal locking occurs when lower order elements such as eight-node hexahedral elements are used to model curved shells so that their cross-sections assume the trapezoidal shape these excessive number of sampled thickness strains can be reduced by using a bilinear interpolation of the transverse normal strains sampled at the four corners of the element mid-surface, namely

$$
\varepsilon_{z}^{A N S}=\sum_{i=1}^{4} N_{i}(\xi, \eta) \varepsilon_{z}\left(\xi_{i}, \eta_{i}\right)
$$

Poisson's ratio coupling requires the thickness strain to be a linear function of $\zeta$. Because our solid-shell element has only two layers, as consequence the thickness strain does not vary with $\zeta$ thus the element fail in reproducing the plane-stress condition. In order to obtain a linear distribution of the normal strain in thickness 
direction, we enhance the thickness strain field by adding an internal degree of freedom

$$
\tilde{\varepsilon}_{z}^{A N S}=\varepsilon_{z}^{A N S}+\alpha \zeta \mathbf{e}_{3}
$$

where $\alpha$ represents the $7^{\text {th }}$ independent internal parameter in the element level which will be eliminated by special condensation technique. Using (12) and (14) into (8), the virtual internal work takes the final expression

$$
W_{i n t}=\sum_{i=1}^{n l} \int_{\xi} \int_{\eta} \int_{\zeta_{i}}^{\zeta_{i+1}}\left(\delta \varepsilon_{s}^{T} \tilde{\mathbf{C}}_{\mathbf{1}} \varepsilon_{s}+\delta \tilde{\varepsilon}_{z}^{A N S^{T}} \tilde{\mathbf{C}}_{\mathbf{2}} \tilde{\varepsilon}_{z}^{A N S}+\delta \gamma_{s}^{A N S^{T}} \tilde{\mathbf{C}}_{\mathbf{3}} \gamma_{s}^{A N S}\right) J d \xi d \eta d \zeta
$$

where $n l$ is the number of layers and $\zeta_{i}$ is the transverse reference coordinate of the $\mathrm{i}^{\text {th }}$ layer along the cross-section of the element. The final stiffness matrix can be obtained after condensation technique to eliminate the internal parameter $\alpha$ by:

$$
\begin{gathered}
\mathbf{K}=\mathbf{K}_{m f}+\mathbf{K}_{e z}^{A N S}+\mathbf{K}_{c}^{A N S}-\beta \mathbf{K}_{\alpha u} \otimes \mathbf{K}_{\alpha u}^{T} \\
\mathbf{K}_{\alpha u}=\sum_{i=1}^{n l} \int_{\xi} \int_{\eta} \int_{\zeta_{i}}^{\zeta_{i+1}} \zeta \tilde{C}_{2}^{33} \mathbf{B}_{z}^{T} \mathbf{e}_{3} J d \xi d \eta d \zeta ; \quad \frac{1}{\beta}=\sum_{i=1}^{n l} \int_{\xi} \int_{\eta} \int_{\zeta_{i}}^{\zeta_{i+1}} \zeta^{2} \tilde{C}_{2}^{33} J d \xi d \eta d \zeta
\end{gathered}
$$

\section{Optimization using RSM based on diffuse approximation}

The optimization problem can be stated as:

$$
\text { minimize } f(\mathbf{x}), \quad \mathbf{x} \in R^{n}
$$

subject to a set of $m+2 n$ constraints

$$
\begin{array}{ll}
g_{j}(\mathbf{x}) \leq 0, & j=1, \ldots, m \\
L_{i} \leq x_{i} \leq U_{i}, & i=1, \ldots, n
\end{array}
$$

where $f$ is the objective function, $x_{i}$ are the design variables, $g_{j}$ is the $j^{\text {th }}$ constraint. The region of interest is defined by $L_{i}$ and $U_{i}$ which are respectively the lower and upper bounds on the design variables. The RSM approach consists in solving a problem where the objective function is replaced by its approximation $\tilde{f}$. This new problem may be written as

$$
\left\{\begin{array}{l}
\text { minimize } \tilde{f}(\mathbf{x}), \quad \mathbf{x} \in R^{n} \\
\text { subject to }(18) .
\end{array}\right.
$$

The approximation (19) is based on a set of numerical experiments with the function $f$. In the actual work, we explore the application of DA for the building of response surface during successive iterations. The approximation is local, what means that only the points closest to the current optimum are taken into account. 
The approximation coefficients are continuous when panning and/or zooming of the region of interest is performed.

Given the function values for a set of experimental points $\mathbf{x}_{i}$ distributed according to a chosen Design of Experiment, the function $\tilde{f}$ can be defined in terms of basis functions $\mathbf{p}$ and some adjusting coefficients $\mathbf{a}$ as

$$
\tilde{f}(\mathbf{x})=\mathbf{p}^{T}(\mathbf{x}) \cdot \mathbf{a}(\mathbf{x})
$$

A common choice for the basis functions $\mathbf{p}$ are linear and quadratic monomials

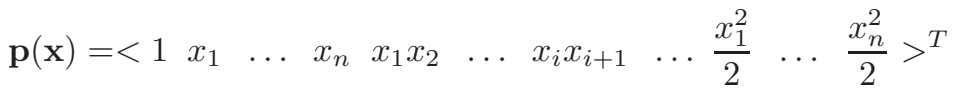

The coefficients of a are determined by a weighted least squares method minimizing the error $J(\mathbf{a})$ between the experimental and approximated values of the objective function

$$
J(\mathbf{a})=\sum_{i=1}^{N} w\left(\left\|\mathbf{x}_{i}-\mathbf{x}\right\|\right)\left(\mathbf{p}^{T}\left(\mathbf{x}_{i}-\mathbf{x}\right) \mathbf{a}-f\left(\mathbf{x}_{i}\right)\right)^{2}
$$

where $N$ is the number of performed experiments and $\mathbf{x}_{i}$ are the experimental designs. The weights $w_{i}$ insure the continuity and the locality of the approximation and are defined $w_{i}>0$, decreasing within a fixed region around the point $i$ called domain of influence of $\mathbf{x}_{i}$ and vanish outside. The weight functions play a crucial role by influencing the way that the coefficients a depend on the location of the design point $\mathbf{x}$. Minimization of $J(\mathbf{a})$ gives

$$
\begin{aligned}
\mathbf{a}(\mathbf{x}) & =\mathbf{A}^{-1} \mathbf{B} \mathbf{f} \\
\mathbf{A} & =\mathbf{P} \mathbf{W} \mathbf{P}^{T} \\
\mathbf{B} & =\mathbf{P} \mathbf{W}
\end{aligned}
$$

Vector a may be interpreted as coefficients of Taylor expansion of $f$ around the evaluation point $\mathbf{x}$. In this sense, a correspond to the approximation of subsequent derivatives - we use the term of "diffuse derivative" and the symbol $\delta$ to differentiate from the "full" derivative $\partial$

$$
\mathbf{a}(\mathbf{x})=<\tilde{f} \frac{\delta f}{\delta x_{1}} \frac{\delta f}{\delta x_{2}} \ldots \frac{\delta f}{\delta x_{n}} \frac{\delta^{2} f}{\delta x_{1} \delta x_{2}} \ldots \frac{\delta^{2} f}{\delta x_{i} \delta x_{i+1}} \ldots \frac{\delta^{2} f}{\delta x_{1}^{2}} \ldots \frac{\delta^{2} f}{\delta x_{n}^{2}}>^{T}
$$

\section{Optimization of a wrapped thick cylinder under pressure}

\subsection{Problem analysis}

The structure is composed of an inner isotropic cylinder $E=210000 M P a$, $v=0.3$ and an outer orthotropic circumferentially wound cylinder 


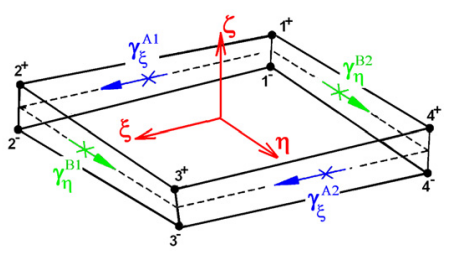

Figure 3: Geometry and loading.

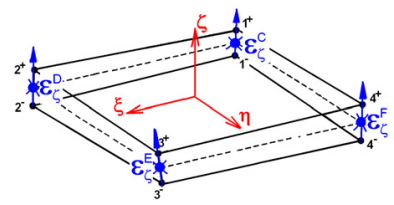

Figure 4: Central composite design.

with: $E_{11}=130000 M P a, \quad E_{22}=E_{33}=5000 M P a, v_{12}=v_{13}=0.25$, $v_{23}=0, G_{12}=G_{13}=10000 M P a$ and $G_{23}=5000 M P a$. The boundary conditions correspond to $u_{z}=0$ at $z=0$ and the cylinder is subjected to an internal pressure of $200 \mathrm{MPa}$ (Figure 3).

First the cylinder is modeled using the initial parameters and a $10 \times 10 \times 1$ solid-shell mesh. The circumferential stress at $\mathrm{z}=0$ is measured for two radius values $(r=23 \mathrm{~mm}, 27 \mathrm{~mm})$ and compared to the NAFEMS reference solution [6] and to the one we obtained using SC8R ABAQUS solid-shell F.E. model. For the inner flange we obtain $\sigma_{11}=1534.8 \mathrm{MPa}$, the SC8R Abaqus gave $\sigma_{11}=1477.0 \mathrm{MPa}$, while the reference solution was $1565 \mathrm{MPa}$. For the outer flange we obtain $\sigma_{11}=892.8 M P a$, the Abaqus solution was $\sigma_{11}=900.0 M P a$, while the reference solution was $875 \mathrm{MPa}$. These results confirm that our model and the used mesh are good enough to carry out the optimization process.

\subsection{Optimization of material parameters}

The optimization problem consists in finding optimal fibber orientation angle $\theta$ of the outer orthotropic circumferentially wound layer and the Young's modulus $E$ of the inner isotropic cylinder while keeping constant the cylinder expansion for $z=0$ at a value of $U_{R}=0.5 \mathrm{~mm}$.

The objective function is based on the general Hill criterion as

$$
\begin{aligned}
J(\theta, E)=J=\sum_{e=1}^{n e l t} & \left(F\left(\sigma_{22}-\sigma_{33}\right)^{2}+G\left(\sigma_{33}-\sigma_{11}\right)^{2}+H\left(\sigma_{11}-\sigma_{22}\right)^{2}\right. \\
& \left.+2 L \tau_{23}^{2}+2 M \tau_{31}^{2}+2 N \tau_{12}^{2}\right)
\end{aligned}
$$

Optimization variables are constrained between $0^{\circ} \leq \theta \leq 90^{\circ}$ and $60 G P a \leq E \leq$ $300 G P a$. At the beginning a Design of Experiments of 9 function evaluations, based on the central composite design algorithm is carried out using three groups of design points ( 4 two-level factorial design points, 4 axial points and a 1 center point). Then design variables are coded as $(-1,+1)$ in order to facilitate the data treatment. The DOE plan with the computed objective function values are given in Table 1.

A global quadratic response surface model based on DA is built using previously prepared experimental designs (see Figure 5). This response function is given explicitly by:

$$
\tilde{J}(\theta, E)=291900+4107.30 \theta+1842.52 E-7520.69 \theta E-10230.38 \theta^{2}-261.44 E^{2}
$$


Table 1: DOE plan using central composite.

\begin{tabular}{c|c|c|c|c}
\hline Run & $\theta$ & E & Response J & Displ. \\
\hline 1 & -1 & -1 & 268316.8 & 0.355 \\
2 & 1 & -1 & 294069.1 & 0.833 \\
3 & -1 & 1 & 282546.0 & 0.150 \\
4 & 1 & 1 & 293785.5 & 0.196 \\
5 & -1 & 0 & 278756.9 & 0.210 \\
6 & 1 & 0 & 294282.9 & 0.316 \\
7 & 0 & -1 & 280609.1 & 0.584 \\
8 & 0 & 1 & 286957.9 & 0.134 \\
9 & 0 & 0 & 285223.1 & 0.211 \\
\hline
\end{tabular}

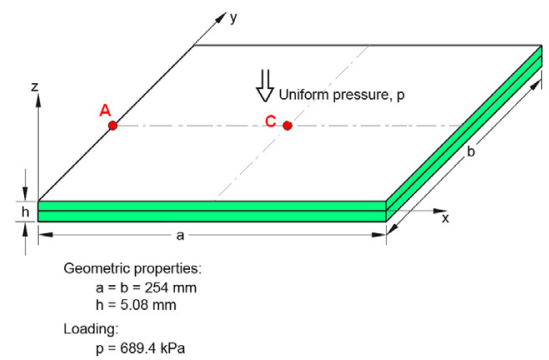

Figure 5: Response surface of objective function.

The response approximation function for the constraint $(U=0.5)$ on the radial displacement of the cylinder at $z=0$ is also carried out using DA based on the data of DOE from Table 1, this $2^{\text {nd }}$ surface is represented on Figure 6 and given explicitly by:

$$
\tilde{U}(\theta, E)=0.49+0.026 \theta+0.071 E+0.13 \theta E-0.14 \theta^{2}-0.098 E^{2}
$$

The minimization of $\tilde{J}(\theta, E)$ under constraint $\tilde{U}(\theta, E)=0.5$ (see Figure 7) has been done using SQP algorithm based on the work of Powell, the optimal solution was obtained in 5 iterations leading to a non symmetric result in coded form $\langle 0.830,0.961\rangle$ which corresponds to

$$
\left\langle\theta_{\text {optimal }}, E_{\text {optimal }}\right\rangle=\left\langle 82.34^{\circ}, 295.40 G P a\right\rangle
$$




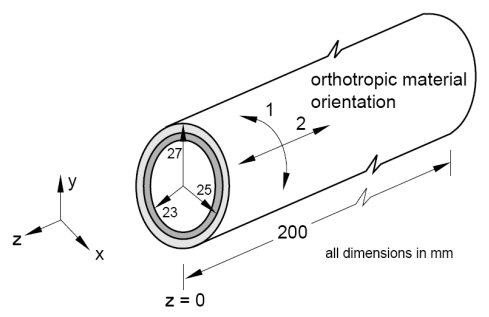

Figure 6: Response surface of constraint function.

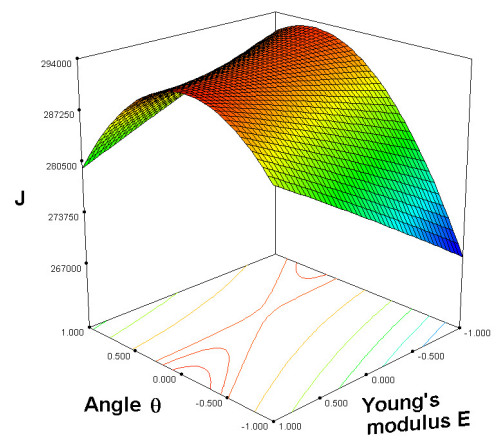

Figure 8: Hoop stress before optimization.

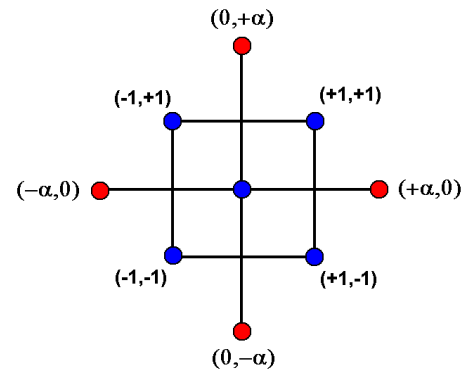

Figure 7: Optimal solution in contour plot.

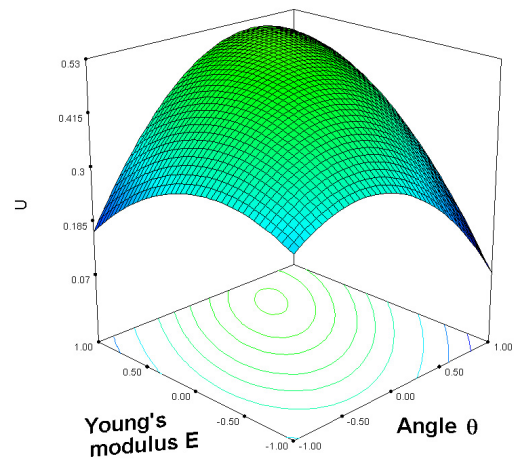

Figure 9: Hoop stress after optimization.

Figure 8 and Figure 9 show the hoop stress distribution on the cylinder before and after optimization. We can observe just a small reduction of stresses after optimization, this is due to the presence of constraint $\tilde{U}(\theta, E)=0.5$ (Figure 7) which can not lead to the minimum of the unconstrained objective function $\tilde{J}(\theta, E)$.

\section{Conclusion}

In this paper, we proposed a specific response surface method based on DA involving pattern search optimization. The resulting response surface algorithm involve iterative improvement of the objective function employing locally supported nonlinear approximations. Numerical examples confirm that the 
optimization method based on response surface and DA coupled to FE solid-shell model is efficient and particularly suited for industrial problems.

\section{References}

[1] Batoz J.L., Dhatt G., 1992. "Modélisation des structures par éléments finis", vol 2, 3, Editions Hermès, Paris.

[2] Domissy E., 1997. "Formulation et évaluation d'éléments finis volumiques modifiés pour l'analyse linéaire et non linéaire des coques", Université de Technologie de Compiègne, 15 mai 1997.

[3] Hauptmann R., Schweizerhof K., 1998. "A systematic development of 'solidshell' element formulation for linear and non-linear analysis employing only displacement degrees of freedom", Int. J. Numer. Meth. Engrg., v.42, pp.49-69.

[4] Klinkel S., Gruttman F., Wagner W., 1999. "A continuum based 3d-shell element for laminated structures", Computers \& Structures, v.71, pp.43-62.

[5] Myers RH, Montgomery DC, 2002. "Response Surface Methodology Process and Product Optimization using Designed Experiments". John Wiley and Sons, Inc., New York, USA, 2nd ed.

[6] National Agency for Finite Element Methods and Standards (NAFEMS), Test R0031/2 from NAFEMS publication R0031, "Composites Benchmarks," Issue 2, Feb. 5, 2001. 